\section{Theoretical \& Applied Science}

p-ISSN: 2308-4944 (print) e-ISSN: 2409-0085 (online)

Year: 2015 Issue: 10 Volume: 30

Published: $30.10 .2015 \quad$ http://T-Science.org
Said Abdullaevich Salekhov

Professor, doctor of medical sciences

Novgorod State University of Yaroslav Mudry, Russia ssalehov@mail.ru

Vladimir Igorevich Esaulov

Assistant professor Department of Psychotherapy, Pirogov Russian National Research Medical University,

Russia

v-esaulov@yandex.ru

Svetlana Olegovna Yablochkina

Candidate of medical sciences, Assistant professor,

Novgorod State University of Yaroslav Mudry, Russia

iablochkina72@mail.ru

\title{
INFLUENCE OF EXPECTANT MOTHERS NEGATIVE STRESS ON COPING STRATEGY AND THEIR ENERGY SUPPLY FORMING
}

\begin{abstract}
The analysis of expectant mother psychological stress influence on the fetus future coping strategies was made in the article. It was based on the principal propositions of psychophysiology, theory of dominant, theory of functional systems, patterns of general adaptation syndrome development, peculiarities of energy support of intense neural activity under psychological stress. The integrative approach to pregnant women psycho-emotional factors, their body reaction to a stressor, ways of informing the fetus about the stress by humoral means of the mother-placenta-fetus system and fetus trophics change influenced by placenta angiospasm together with sympathetic-adrenal system activation, let us specify two directions of coping strategy forming. On one side, mother's negative emotions and her humoral factors, forming her psycho-emotional condition, pass to the fetus by the mother-placenta-fetus system the information about stress impact, the analogy of which is an unmet need. Depending on mother's coping strategies and reaction effectiveness to the stress impact, the change of her psychological state will show either regress of stress intensity, that is of the need satisfaction followed by positive emotional reinforcement, or of the persistent unmet need and negative emotional reinforcement. On the other side, the strategies, which provide energy supplies to organism stress reaction, are equally important. If mother's coping strategies are effective, no significant changes of the fetus trophics will occur. On the contrary, if mother's stress is long enough and the changes of the fetus trophics are significant, either nutritional behavior "stress - hunger stress-eating" of "stress - hunger - self-eating" will form. As a result, mother's stress during pregnancy makes preconditions to future coping strategies and nutritional behavior.
\end{abstract}

Key words: psychological stress, pregnancy, theory of dominant, theory of functional systems, coping strategies, nutritional behavior, unmet needs, negative emotions, need satisfaction, positive emotional reinforcement.

Language: Russian

Citation: Salekhov SA, Esaulov VI, Yablochkina SO (2015) INFLUENCE OF EXPECTANT MOTHERS NEGATIVE STRESS ON COPING STRATEGY AND THEIR ENERGY SUPPLY FORMING. ISJ Theoretical \& Applied Science 10 (30): 111-116.

Soi: http://s-o-i.org/1.1/TAS-10-30-24 Doi: crossef http://dx.doi.org/10.15863/TAS.2015.10.30.24

\section{ВЛИЯНИЕ НЕГАТИВНОГО СТРЕССА БЕРЕМЕННЫХ НА ФОРМИРОВАНИЕ КОППИНГ- СТРАТЕГИЙ И ИХ ЭНЕРГЕТИЧЕСКОГО ОБЕСПЕЧЕНИЯ}

Аннотация: В статье, опираясь на фундаментальные положения психофизиологии, учения о доминанте, теории функциональных систем, закономерности развития общего адаптационного синдрома, особенности энергетического обеспечения интенсивной нервной деятельности при психологической нагрузке был проведен анализ влияния психологического стресса у беременной на формирование коппингстратегий и пищевого поведения у плода, реализующихся в последующей жизни. Интегративный подход в рассмотрении психоэмоциональных факторов у беременной, реакции ее организма на воздействие стрессора, передача информации о стрессе плоду гуморальным путем по системе мать-плацента-плод и изменения трофики плода под воздействием ангиоспазма сосудов плаценты на фоне активации симпатоадреналовой системы позволил выделить два вектора в формировании коппинг-стратегий. $C$ одной стороны, негативные эмоции у матери с гуморальными факторами, определяющцими ее 
психоэмоцииональое состояние, передают плоду, по системе мать-плацента-плод, информацию о стрессовом воздействии, аналогом которого является неудовлетворенная потребность. В зависимости от коппинг-стратегий матери и эффективности реакичии на воздействие стрессора изменение ее психологического состояния будет свидетельствовать либо о регрессии интенсивности стресса, то есть об удовлетворении потребности, что проявляется положительным эмоцииональным подкреплением, либо о сохранении состояния неудовлетворенной потребности и негативном эмоциональном подкреплении. Не менее важным является стратегии обеспечивающей энергетическое обеспечение реакции организма на стресс. При эффективных коппинг-стратегиях матери существенных изменений в трофике плода происходить не будет. В отличие от этого, в зависимости от продолжительности стресса у матери, при выраженных изменениях трофики плода будет формироваться пищевое поведение «стресс-голод-заедание стресса», либо «стресс-голод-самопожирание». Таким образом, стресс во время беременности у матери создает предпосылки для формирования коппинг-стратегий и пищевого поведения, которые могут реализоваться в будущем.

Ключевые слова: психологический стресс, беременность, принции доминанты, теория функциональных систем, коппинг-стратегии, пищевое поведение, неудовлетворенные потребности, отрицательные эмоции, удовлетворенные потребности, положительное эмоциональное подкрепление.

Стресс стал постоянным спутником современного образа жизни. В отличие от жизни первобытного человека, в процессе эволюции на смену реально угрожающим жизни эпизодам, как правило, кратковременным, пришло ожидание ситуаций, которые воспринимаются аналогичным образом без угрозы жизни и здоровью $[1$, с. 88 92; 2, с. 70-71]. Так, на смену страху, который исчезает после устранения угрозы жизни и здоровью пришла тревога, появляющаяся задолго до ситуации, воспринимаемой как стрессовая и часто сохраняющаяся длительное время после ее разрешения.

По сути, раньше в ответ на воздействие стрессора следовал немедленный ответ, направленный на удовлетворение его запроса и нейтрализацию негативных последствий. Воздействие стрессора активизировало симпатоадреналовую систему, следствием которой была реакция «борьба-бегство» [1, с. 21 22], направленную на поддержание гомеостаза [3].

В отличие от этого типа реагирования, при стрессах, сопутствующих современному образу жизни, мобилизация организма часто происходит задолго до воздействия стрессора [4], и предполагает готовность к самым негативным последствиям. Более того, ожидаемая стрессовая ситуации, а тем более ее негативных последствий могут просто не случиться. При этом на удовлетворение длительной готовности реакции на воздействие стрессора, триггером которой стала тревожность, расходуются компенсаторноприспособительные ресурсы организма, что создает предпосылки к их истощению.

Следует отметить, что вне зависимости от вида стрессора, возникающие ответные реакции сопровождаются как изменением эмоционального состояния, так и параллельно возникающими изменениями физического состояния [5, с. 72$]$.
Основное внимание в настоящее время уделяется роли центральной нервной системы (ЦНС), являющейся основной мишенью для воздействия стрессора. При этом происходит постепенная смена приоритетности биологического компонента стресса на психологический, сфокусированного как на психоэмоциональной, так и когнитивной сфере деятельности ЦНС [6, с. 37].

Использование когнитивной функции позволяет выбрать более эффективную реакцию на воздействие психологического стрессора, а психоэмоциональная реакция на него может привести к блоку когнитивного осмысления стрессовой ситуации и реагирования на нее по типу «борьба-бегство».

По мнению Lazarus R.S. (1977), эмоции, в сочетании c когнитивной функцией, имеют большое значение в формировании коппингстратегий при воздействии стрессора [7].

Следует отметить, что по мере развития организма сначала закладываются паттерны реагирования на воздействие стрессора в виде стратегий предусматривающих реакцию «борьбабегство» и только после этого, значительно позднее происходит обучение и встраивание в коппинг-стратегии когнитивного осмысления ситуации, дающего конкурентные преимущества в условиях постоянно меняющегося современного мира.

Первый опыт общения с миром организм будущего человека приобретает от момента зачатия до рождения, опираясь на информацию, опосредованно получаемую от матери. Именно анализу этого периода развития организма и его влиянию на формирование коппинг-стратегий посвящено это сообщение.

Целью нашего исследования являлось теоретическое обоснование значения стресса во время беременности на формирование коппингстратегий плода и энергетического обеспечения их реализации. 


\section{Материалы и методы исследования}

Обоснование особенностей влияния стресса во время беременности на формирование коппинг-стратегий и энергетического обеспечения их реализации проводили, опираясь на учение о доминанте [8], теорию функциональных систем [9], закономерностей развития общего адаптационного синдрома [10], физиологические изменения энергетического обеспечения мозга при психологическом стрессе [6].

Принцип доминанты, описанный А.А. Ухтомским гласит, что возникновение очага приоритетного, доминирующего возбуждения в ЦНС приводит к перераспределению энергетических и пластических ресурсов в пользу удовлетворения этого доминантного очага возбуждения. Соответственно, потребности остальных отделов ЦНС обеспечиваются в ограниченном количестве, по остаточному принципу, что приводит к снижению их функциональной эффективности [8].

При воздействии на организм стрессора формируется функциональная система с целью достижения конечного положительного результата, и поддержания гомеостаза [9]. Это обеспечивается адекватной ответной реакцией организма на стрессовую ситуацию с привлечением компенсаторноприспособительных ресурсов организма и в соответствии с индивидуальными коппингстратегиями. Структура и последовательность деятельности функциональной системы заключается в том, что:

-при поступлении входящей информации аппарат контроля формирует модель полезного результата;

-аппарат управления, объединяющий нервные центры и эндокринные органы определяет необходимые ресурсы для достижения полезного результата;

-исполнительный аппарат, представленный органами-аффекторами, используя выделенные ресурсы, обеспечивает реализацию получения результата;

-аппарат контроля, по принципу обратной связи тестирует полученный результат на соответствие модели полезного результата.

Если соответствие достигнуто функциональная система завершает свою работу, а если нет, функциональная система вырабатывает новую стратегию достижения модели полезного результата с привлечением новых ресурсов организма. Цикл достижения модели полезного результата и обратной связи повторяется снова до получения соответствия полученного результата модели конечного положительного результата, запрограммированного центром контроля, либо истощения ресурсов организма и декомпенсации [6, с. 45-54; 9, с. 19-54].

В основу теории о стрессе были положены наблюдения H.Selye, установившего, что на любое интенсивное воздействие организм реагирует одинаково, развитием общего адаптационного синдрома [10].

В своей концепции биологической природы эмоции как продукта эволюции П. К. Анохин (1964) рассматривал возникновение потребностей как причину появления негативных эмоций, которые приводили к мобилизации компенсаторно-приспособительных ресурсов организма для удовлетворения конечного положительного результата - удовлетворения потребностей. Когда конечный положительный результат достигнут, а потребность удовлетворена возникают положительные эмоции, которые в виде обратной связи являются триггером для прекращения деятельности данной функциональной системы. Неоднократное удовлетворение потребностей, окрашенное положительной эмоцией, способствует обучению соответствующей деятельности, а повторные неудачи в получении запрограммированного результата вызывают торможение неэффективной деятельности и поиски новых, более успешных способов достижения цели. [11, c. 339].

То есть, по сути своей, возникновение отрицательных эмоций является маркером стрессового состояния, а после ее разрешения положительные эмоции свидетельствуют об эффективной реакции организма на воздействие стрессора и достижения конечного положительного результата в виде удовлетворения запроса стрессора и сохранении гомеостаза в данный текущий момент времени.

На фоне интенсивной деятельности ЦНС отмечается увеличение потребления мозгом кислорода и глюкозы, что обеспечивает удовлетворение возрастающего запроса в энергии [6, с. 126-136]. При этом недостаток в поступлении либо кислорода, либо глюкозы, обеспечивающий аэробный катаболизм глюкозы в нервных клетках сопровождается энергетическим дефицитом, следствием чего будет торможение. То есть, при воздействии стрессора эффективность реакции на него с одной стороны будет зависеть от коппингстратегии, усвоенной на основании предыдущего опыта, в частности во время беременности, а с другой от энергетического обеспечения реализации этой коппинг-стратегии, в первую очередь регуляторной функции ЦНС. 
Обоснование концепции формирования коппинг-стратегий и энергетического обеспечения стресса

В течение всей беременности состояние плода зависит от состояния здоровья и психоэмоционального состояния будущей матери. Характер поступающей информации от беременной к плоду будет обусловлен реакцией ее организма на внешние и внутренние факторы тем "коктейлем медиаторов", который будет отражать ее реакцию на течение беременности.
Следует отметить, что психоэмоциональное состояние беременных является достаточно лабильным, а реакции на раздражители могут быть парадоксальными. Так, реакция на сильный, в других условиях раздражитель может быть минимальны, а при минимальных воздействиях, напротив, вызывать бурную интенсивную реакцию.

Схема влияния негативного стресса беременных на формирование коппинг-стратегий и их энергетического обеспечения может быть представлена в следующем виде (рис.1)

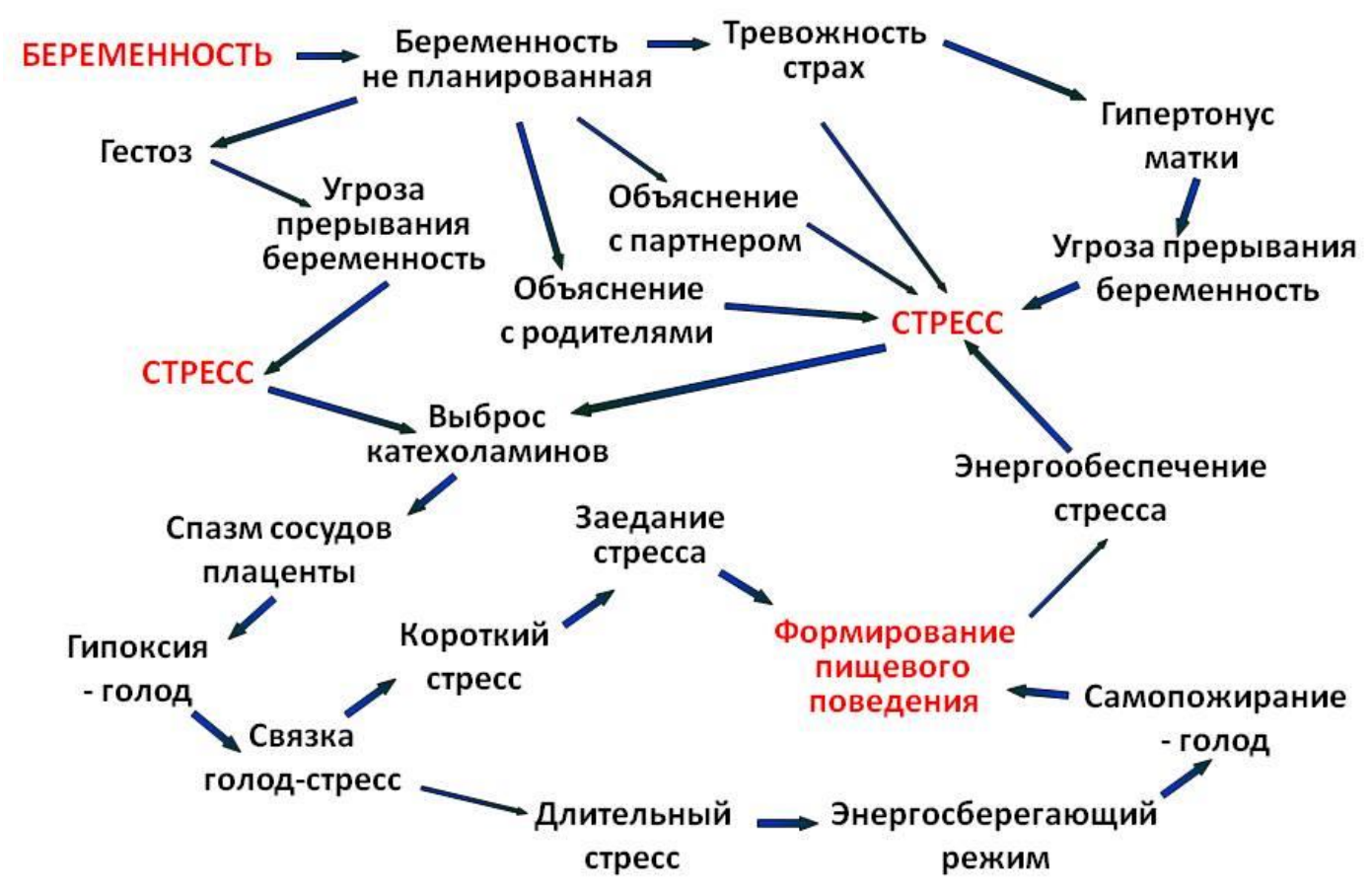

\section{Рисунок 1 - Влияние стресса беременных на формирование коппинг-стратегий и их энергетического обеспечения.}

Большое значение имеет первая реакция женщины, узнавшей о беременности. Здесь можно отметить целый комплекс факторов, оказывающих влияние на психоэмоциональное состояние.

В данной ситуации определенную роль может играть социальный статус на момент начала беременности (замужество, гражданский брак или случайная связь). Кроме того, важными факторами являются значение беременности для женщины: желанная ли беременность, длительно ожидаемая, случайная или незапланированная, после насильственного полового акта.

Так, любой из вариантов незапланированной беременности, начиная с момента ее диагностики/самодиагностики, является стрессом и сопровождается психоэмоциональным напряжением, интенсивность которого будет зависеть от индивидуальной истории женщины, ее личностных особенностей. Большое значение имеет изменения отношений с отцом будущего ребенка, его реакцией на известие о беременности.

Совокупность большого числа факторов будет определять результат решения о сохранении или прерывании беременности. На этом фоне появляются повышенная тревожность, раздражение, агрессия, плаксивость, нарушение сна, страх перед разговором с родителями, объяснения с партнером, первая реакция которых может быть достаточно непредсказуемой (вплоть до агрессии), даже если в дальнейшем будет принято решение о сохранении беременности и ее принятии с их стороны.

При этом психоэмоциональный стресс беременной оказывает негативное влияние на 
плод еще на стадии закладки органов и систем. Сохраняющиеся длительное время отрицательные эмоции являются маркером стрессовой ситуации, когда потребности плода не удовлетворяются. Поскольку сам он их удовлетворить не в состоянии, а зависит от состояния организма матери и ее эмоционального фона, динамика гуморальных факторов, поступающих через систему мать-плацента-плод и реакция на них организма будут определять формирование коппинг-стратегий.

На фоне психоэмоционального стресса происходит активация симпато-адреналовой системы, следствием чего является выброс катехоламинов, что приводит к периферическому спазму сосудов, гипертонусу миометрия, спазму мышц различных отделов пищеварительного тракта. Таким образом, создаются предпосылки для дефицита трофики яйцеклетки, аналогом чего является голод.

Кроме того, часто сопровождающие стресс нарушения сна сопровождаются снижением восстановительных процессов, утилизации и элиминации токсичных метаболитов, что, в свою очередь приводит к эндогенной интоксикации и, опосредовано, к нейротоксикозу.

Учитывая, что совокупность гуморальных факторов при позитивном и негативном состояниях отличаются друг от друга, на подсознательном уровне у будущего ребенка уже на этом этапе может закладываться отверженность, отсутствие любви и принятия.

В дальнейшем каждый эпизод психоэмоционального стресса во время беременности, любая патология ее течения, так же имеющая психоэмоциональную окраску может оказывать отрицательное влияние на беременную и, опосредовано на плод. Дефицит внимания, воспринимаемый беременной как одиночество, отсутствие помощи от окружающих, беззащитность, беспомощность автоматически передается плоду, так же как и характерные для нее коппинг-стратегии в этих ситуациях. При этом моделирование реакций на те или иные воздействия во время беременности будут закрепляться на уровне рефлексов, а в дальнейшем реализовываться при появлении стрессовых ситуаций.

Реакция организма на психологический стресс во время беременности оказывает на плод двойное действие. С одной стороны он получает информацию, обусловленную поступление гуморальных факторов отражающих и моделирующих состояние матери по системе «мать-плацента-плод», a c другой реакция сосудистого микроциркуляторного русла плаценты на выброс катехоламинов приводит к снижению плацентарного кровотока, тем самым создавая дефицит поступления питательных веществ.

На этом фоне формируется связка «катехоламины-голод». Повторение стрессовых ситуаций будет закреплять эту связку. То есть формируются предпосылки для усиленного потребления пищи при выбросе катехоламинов, поскольку закреплен рефлекс «катехоламиныголод».

Для формирования готовности к учащенному употреблению пищи определенное значение может иметь и продолжительность стресса у беременной, вне зависимости от ее пищевого поведения в стрессовой ситуации. У плода энергетическое обеспечение стресса будет формироваться от его индивидуальной реакции на дефицит поступления энергоносителей на фоне снижения плацентарного кровотока. Так, при кратковременном стрессе, после которого быстро восстанавливается плацентарный кровоток будет формироваться паттерн заедания стресса - «стресс-заедание».

В отличие от этого, при длительном стрессе, а соответственно, длительном дефиците поступления питательных веществ к плоду, согласно принципу доминанты, будут блокироваться энергозатратные процессы, а энергетическое удовлетворение запроса стрессора к организму будет происходить за счет использования внутренних ресурсов - «стрессблок аппетита-самопожирание».

В данной ситуации будет два варианта формирования пищевого поведения:

1. Постстрессовое заедание, направленное на восстановление ресурсов и формирование запаса энергоносителей с учетом вероятности стрессовых ситуаций в будущем.

2. Сохранение режима дефицитарного обеспечения энергозатратных процессов в организме, сохраняя готовность к быстрому реагированию на воздействие стрессоров.

Различные негативные психоэмоциональные состояния, обусловленные стрессом [12, р. 1427; 13, p. 594; 14, p. 109], создают предпосылки для изменения пищевого поведения и развития метаболических нарушений [6, с. 139-145; 12, р. 1427-1428; 15], следствием чего являются повышение артериального давления, сердечнососудистые заболевания [13, р. 597-598; 16, р. 289-300], инсулинорезистентность [6, с. 146-131] и гипергликемия [6, с. 160-162]. То есть, на фоне стресса происходит декомпенсация компенсаторно-приспособительных ресурсов, что приводит к развитию различных заболеваний [17, p. 600-601]. По сути своей, это отражает особенности реагирования на психоэмоциональный стресс и энергетического обеспечения этой реакции, первым шагом 
формирования которых является информация заложенная во внутриутробном периоде.

Таким образом, стрессовые ситуации во время беременности с одной стороны создают предпосылки усвоения коппинг-стратегий, основанных на опыте совладания со стрессом матери, а с другой энергетическое обеспечение реализации коппинг-стратегий на воздействие стрессора будет происходить в зависимости от особенностей трофики плода во время стрессовых ситуаций у беременных. При этом на фоне стрессовых ситуаций энергетическое обеспечение стресса найдет отражение в особенностях пищевого поведения при воздействии стрессора.

\section{References:}

1. Grinberg JS (2002) Upravlenie stressom. S-Pb, "Piter", 2002, - 496 p. (Seriya Mastera psihologii)

2. Sudakov KV (1997) Psihoehmocionalnyj stress : profilaktika i reabilitaciya. //Terapevt.Arh. T 69, No 1. 1997, - pp.70-74.

3. Cannon W (1932) The wisdom of the body. N.Y., 1932,- 273 p.

4. Salekhov SA, Gordeev MN, Bizina TV, Maksimyuk NN (2015) Ierarhiya psihoehmocionalnogo i informacionnogo faktorov v strukture ehkzamenacionnogo stressa // Sovremennye problemy nauki i obrazovaniya. No 5, 2015 / Available: http://www.scienceeducation.ru/128-21959 (Accessed: 05.10.2015)

5. Ilyin EP (2001) Emocii i chuvstva. S-Pb. "Piter", 2001, - 752 p. (Seriya Mastera psihologii)

6. Salekhov SA (2014) Psihoehmocionalnaya informacionno ehnergeticheskaya teoriya ozhireniya. Velikij Novgorod-Almaty, 2014.$180 \mathrm{p}$.

7. Lazarus RS (1977) Cognitive and coping processes in emotion. / / Stress and coping. N.Y., Columbia Univ. Press, 1977, pp. 144-157.

8. Uhtomskij AA (1923) Dominanta kak rabochij princip nervnyh centrov /AA Uhtomskij // Russk.fiziol.zhurn., T.VI, vyp. 1-3, 1923, pp. 31-45.

9. Anohin PK (1970) Teoriya funkcionalnoj sistemy / PK Anohin // Uspekhi fiziol. nauk. T.1, No 1, 1970, pp. 19-54

10. Selye HA (1936) Syndrome produced by Diverse Nocuous Agents. // Nature 138, 32 (4 July 1936) doi:10.1038/138032a0

11. Anohin PK (1964) EHmocii // Bolshaya medicinskaya ehnciklopediya. T.35, Moscow, 1964, pp.339.

12. Pagoto S (2013) Randomized controlled trial of behavioral treatment for comorbid obesity and depression in women: the Be Active Trial / S
Pagoto, K L Schneider, M C Whited, J L Oleski, P Merriam, B Appelhans, Y Ma, B Olendzki, M E Waring, A M Busch, S Lemon, I Ockene, S Crawford // International Journal of Obesity No 37, (November, 2013) - pp.14271434 doi:10.1038/ijo.2013.25

13. Ziv A (2013) Comprehensive Approach to Lower Blood Pressure (CALM-BP): a randomized controlled trial of a multifactorial lifestyle intervention / A. Ziv, O. Vogel, D. Keret, S. Pintov, E. Bodenstein, K. Wolkomir, K. Doenyas, Y Mirovski, S. Efrati // J Hum. Hypert. No 27, 2013,- pp.594-600; doi:10.1038/jhh.2013.29 published online 18 April 2013.

14. Newbury-Birch D (2001) Psychological stress, anxiety, depression, job satisfaction, and personality characteristics in preregistration house officers. / Newbury-Birch D., Kamali F. // Postgrad Med J. Feb;77(904): 2001, -pp.109111.

15. Jääskeläinen A (2015) Association between occupational psychosocial factors and waist circumference is modified by diet among men / A Jääskeläinen, L Kaila-Kangas, P Leino-Arjas, M-L Lindbohm, N Nevanperä, J Remes, M-R Järvelin, J Laitinen // European Journal of Clinical Nutrition, (22 April 2015) doi:10.1038/ejcn.2015.59

16. Brent M (2015) Prehypertension - prevalence, health risks, and management strategies / Brent M. Egan, S. Stevens-Fabry // Nature Reviews Cardiology. No 12, 2015, pp.289-300 doi:10.1038/nrcardio.2015.17

17. Brame AL (2010) Stressing the obvious? An allostatic look at critical illness. / Brame A.L., Singer M. // Crit Care Med. Oct; 38, 2010 (10 Suppl):2010 -pp.600-607. doi: 10.1097/CCM.0b013e3181f23e92 